\title{
Neurotoxins from Plectreurys Spider Venom are Potent Presynaptic Blockers in Drosophila
}

\author{
W. D. Branton, L. Kolton, Y. N. Jan, and L. Y. Jan \\ Howard Hughes Medical Institute and Department of Physiology, University of California, San Francisco, California 94143
}

\begin{abstract}
Studies of presynaptic events in synaptic transmission may be facilitated through the use of specific ligands for functional components of the transmitter release mechanism and through the use of genetics. For this purpose, neurotoxins that affect neuromuscular transmission in Drosophila have been identified and purified from Plectreurys spider venom (PLTX). One class of toxins causes irreversible presynaptic block, probably by blocking calcium entry or by acting on other closely associated processes. These toxins have been highly purified and are peptides of about $7 \mathrm{kDa}$ in molecular weight. They specifically block transmitter release at nanomolar concentrations and may be useful in further biochemical studies.
\end{abstract}

Potent biological toxins have been important in the isolation and charactcrization of molecules involved in neurotransmission, such as sodium channels (Agnew, 1984; Catterall, 1986; Noda et al., 1986) and acetylcholine receptors (Noda et al., 1983; Anholt et al., 1984). Many other components of neurotransmission, however, have not been characterized biochemically. Toxins that block certain potassium (Hughes et al., 1982; Miller et al., 1985; Halliwell et al., 1986) and calcium (Kerr and Yoshikami, 1984; Olivera et al., 1985) conductances in vertebrates have been found, and they may be useful tools in biochemical as well as physiological analyses. Similar studies in Drosophila melanogaster could be further facilitated by the use of mutants, and by the use of transformants carrying mutagenized genes. To identify neurotoxins that are potent in Drosophila, we screened commercially available venom of various spider species. We were particularly interested in venoms containing toxins that might be good ligands for previously uncharacterized synaptic membrane channels or receptors. The venom of the spiders Agelenopsis aperta, Hololena curta, and Plectreurys tristes all produced essentially irreversible presynaptic block at Drosophila larval neuromuscular junction. Only Hololena and Plectreurys venoms were available to us in quantities sufficient for detailed biochemical and physiological studies. Some of the toxins

\footnotetext{
Received Mar. 20, 1987; revised June 3, 1987; accepted June 3, 1987

This work is supported by the Howard Hughes Medical Institute and NIH Grant 15963 . We would like to thank Dr. Leslie Timpe, who did the voltageclamp of pupal flight muscle, and Dr. Heidi Phillips, who did the anatomical binding, for allowing us to use their preliminary findings in the Discussion. We also thank Chuck Kristensen at Spider Pharm and Dr. Hunter Jackson at Bio Actives for their help and advice concerning venoms, Pamela Lee for help in assaying materials for activity, Dr. Barry Rothman for advice on HPLC gel filtration, and Phyllis Cameron and Lisa Schulte for expert help in preparation of the manuscript.

Correspondence should be addressed to Dale Branton, Howard Hughes Medical Institute, 3rd \& Parnassus, P.O. Box 0724, San Francisco, CA 94143.

Copyright $(\mathcal{C}) 1987$ Society for Neuroscience $0270-6474 / 87 / 124195-06 \$ 02.00 / 0$
}

from Hololena have been characterized separately in a parallel study (Bowers et al., 1987). We found in the venom of the spider Plectreurys tristis inhibitory and excitatory neurotoxins with essentially irreversible actions on the Drosophila larval neuromuscular junction. We have purified inhibitory toxins that produce rapid and complete block of synaptic transmission at nanomolar concentrations. They may be potent blockers of calcium entry or other processes involved in transmitter release.

\section{Materials and Methods}

Venom. Crude Plectreurys venom (PLTX) was purchased from Spider Pharm, Black Canyon City, AZ, and from BioActives, Salt Lake City, UT. The venom was produced by electrical milking procedures from collected and laboratory-reared spiders, and was specially selected to be free of regurgitated digestive juices.

Electrophysiology. Standard electrophysiological techniques were used to record from the Drosophila larval neuromuscular junction, as previously described (Jan and Jan, 1976). The physiological saline contained $120 \mathrm{~mm} \mathrm{NaCl}, 2 \mathrm{~mm} \mathrm{KCl}, 4 \mathrm{~mm} \mathrm{MgCl}, 0.5 \mathrm{~mm} \mathrm{CaCl}, 36 \mathrm{~mm}$ sucrose, and $5 \mathrm{~mm}$ HEPES buffer, $\mathrm{pH}$ 7.3. The nerve innervating a single hemisegment was drawn into a stimulating suction electrode, and one of the longitudinal muscles of the hemisegment was impaled with a $20 \mathrm{M} \Omega, 3 \mathrm{M} \mathrm{KCl}$-filled glass microelectrode. The nerve was stimulated once every $5 \mathrm{sec}$ (except where otherwise noted) with a $0.2 \mathrm{msec}$ pulse at twice threshold for activation. Methods for stimulating and recording the excitatory junction potential (ejp) in the experiments with mutant larvae (Figs. 4,5 ) were the same, except that the saline contained 0.15 mм CaCl. Extracellular recordings (Figs. 4,5 ) were made from a small loop of motor nerve pulled into a tight-fitting glass suction electrode at a distance of about $200 \mu \mathrm{m}$ from the nerve terminals (Wu et al., 1978). Toxins were applied with vigorous mixing to the bathing solution via small aliquots from concentrated stock solutions. Concentrations of toxins used in physiological studies were estimated by comparing the absorbance peak at $214 \mathrm{~nm}$ on high-performance liquid chromatography (HPLC) gel filtration to that of insulin. The validity of these estimates for alpha-PLTX II, the most abundant toxin found, has been corroborated by preliminary amino acid analysis and microsequence data.

Purification of toxins. All chromatography was done with a Waters HPLC system. Water and acetonitrile (ACN) were HPLC grade. Trifluoroacetic acid (TFA) and heptafluorobutyric acid (HFBA) were Pierce Sequanal reagents. Effluents were monitored for absorbance at $214 \mathrm{~nm}$. In the region of absorbance peaks, $0.2 \mathrm{ml}$ fractions were collected, assayed for activity as in Figure 1, and active regions (Fig. 2, underline) were pooled and lyophylized for further study. Initial HPLC gel filtration was done with $7.5 \times 300 \mathrm{~mm}$ TSK Bio Sil 125 and 250 columns (BioRad) in series with each other, and a $7.5 \times 75 \mathrm{~mm}$ precolumn. The mobile phase was $32 \% \mathrm{ACN}, 0.1 \% \mathrm{TFA}$, flow rate $1.0 \mathrm{ml} / \mathrm{min}$. Injection volume was $20 \mu \mathrm{l}$. For reverse phase, appropriate fractions from gelfiltration runs were pooled, lyophylized, and resuspended in $0.1 \%$ TFA. This material was loaded onto a $4.6 \times 25 \mathrm{~cm} \mathrm{C1} 8$ reverse-phase column (Vydac TP218), equilibrated with $0.1 \%$ TFA, and the column eluted at a flow rate of $1.0 \mathrm{ml} / \mathrm{min}$ with a linear gradient of $0-60 \% \mathrm{ACN}(0.1 \%$ TFA) in $60 \mathrm{~min}$. Inhibitory peak II (alpha-PLTX II) was repurified on the same reverse-phase column under isocratic conditions (approximately $45 \% \mathrm{ACN}$ ) using $0.05 \%$ HFBA in place of TFA, and was finally repurified on 2 TSK 250 gel-filtration columns in series without a precolumn. 

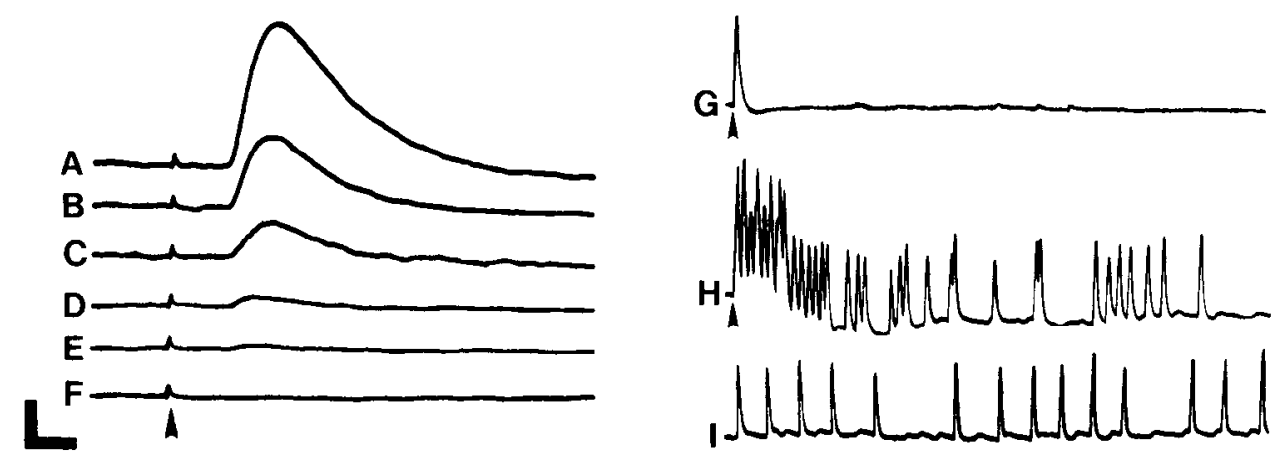

Figure 1. Inhibitory and excitatory activities found in the Plectreurys spider venom. The effect of the inhibitory toxin on excitatory junction potentials (ejps) at the Drosophila larval neuromuscular junction is shown in oscilloscope tracings $A-F$. $A$, Control trace in the absence of toxin. $B-F$, Sequential traces taken at $1 \mathrm{~min}$ intervals after the application of $10^{-9} \mathrm{M}$ alpha-PLTX II. The block of transmission is progressive and complete within 5 min. $G-I$, Effect of the excitatory activity on the larval neuromuscular preparation. $G$, Control tracc showing a single ejp evoked in response to a single nerve stimulus. $H$ and $I$, Traces taken $10 \mathrm{~min}$ after the application of the excitatory toxin at a dilution of $10^{4}$ relative to crude venom. In $H$ there is a burst of ejps following the same stimulus delivered in $G$. $I$, Spontaneous activity in the absence of electrical stimulation. Vertical calibration bar, $5 \mathrm{mV}$. Horizontal calibration bar: $5 \mathrm{msec}(A-F) ; 0.2 \sec (G-I)$.

We have included analytical traces of PLTX under isocratic conditions in Figure 2 in order to indicate the apparent purity that we have achieved, but the retention time under these conditions can vary greatly with the condition of the column and precolumn used, the precise concentration of $A C N$ and buffer in the mobile phase, and the amount of material loaded on the column. Generally we have found it necessary to verify the toxin peak at each step by bioassay on larval neuromuscular junction.

\section{Results}

The Drosophila larval neuromuscular junction preparation (Jan and Jan, 1976) was used in analyzing the action of neurotoxins from spider venoms (Figs. 1, 3, 4, 5). A single suprathreshold stimulus to the segmental nerve normally gives a single compound action potential (Wu et al., 1978). The action potential in the nerve, in turn, gives rise to a single excitatory junctional potential (ejp) in the muscle. Spontaneous miniature ejps (mejps) are easily observed with intracellular recording of the postsynaptic muscle cell. When axonal conduction is blocked by addition of TTX, an ejp can still be generated if the length of the nerve between the stimulating electrode and the muscle is reduced to a few hundred microns, thereby allowing direct stimulation of the nerve terminal. This normal pattern of synaptic transmission was altered by neurotoxins present in the spider venom. Crude Plectreurys venom contains many different components that interfere with Drosophila neuromuscular transmission. Although we have not exhaustively ruled out possible secondary effects of the toxins described in this report, they were chosen for study because their effects appear to be specific, potent, and not easily reversible.

The excitatory effect of the venom was manifested by multiple ejps in response to a single stimulus (Fig. $1 H$ ) and by the occurrence of frequent spontaneous ejps in the absence of nerve stimulation (Fig. 1I). These effects were not easily reversible; they persisted after the recording chamber was washed for an hour with toxin-free saline. This activity had an apparent $M_{\mathrm{r}}$ of $8-10 \mathrm{kDa}$ and was largely confined to a peak of material identified on reverse-phase HPLC gradients (Fig. $2 A, B$ ). Its effects were similar to those of scorpion venoms on the same preparation, and were completely abolished by TTX under conditions where ejps could still be elicited by direct stimulation of the nerve terminal. Therefore, the excitatory effects were presumed to be caused by an action on axonal sodium or potassium channels, and have not been studied further.

The inhibitory activity was of particular interest because its effects were fully consistent with a specific and irreversible block of presynaptic calcium channels. The activity had a $M_{\mathrm{r}}$ of about 6-7 kDa (Fig. $2 A, E$ ) and was confined to a restricted region of reverse-phase HPLC gradients (Fig. $2 C$ ). Three particularly potent activities were identified in this region and the toxins designated alpha-Plectreurys toxin (PLTX) I, II, and III. All 3 were purified. They showed an identical action on the neuromuscular junction. Alpha-PLTX II has been the most consistently abundant toxin in various samples of venom and conscquently has been studied more thoroughly.

Purification of alpha-PLTX II was achieved in 4 steps: After HPLC gel filtration (Fig. $2 A$ ) and reverse-phase gradient (Fig. $2 C$ ), a second solvent system was used to rechromatograph the toxin on reverse-phase columns, and final purification was carried out on a high-resolution gel-filtration column. Analytical chromatograms of the final fraction on reverse phase (Fig. 2D) and gel filtration (Fig. $2 E$ ) showed a single, sharp peak of absorbance, corresponding to the biological activity of the toxin. This toxin appeared to be composed of a single polypeptide, because reduced and alkylated toxin ran as a single peak on HPLC gel filtration (not shown), with an apparent $M_{\mathrm{r}}$ of $7 \mathrm{kDa}$. Preliminary amino acid analysis and microsequence data have further corroborated that the purified toxin is a polypeptide of about $7 \mathrm{kDa}$. Alpha-PLTX II represented about $0.1 \%$ of total protein in crude venom, and accounted for about $20 \%$ of the neuromuscular blocking activity of the venom.

The purified toxin has very potent and specific physiological effects. When alpha-PLTX II was applied at a nanomolar concentration in the saline bathing the neuromuscular preparation, the ejp gradually disappeared over a period of about $5 \mathrm{~min}$ (Fig. $1, A-F)$. The action of the toxin was irreversible; the block could not be reversed by perfusing the chamber with saline for up to $2 \mathrm{hr}$. At higher doses of toxin $\left(10^{-8} \mathrm{M}\right)$, the ejp could be blocked in less than $1 \mathrm{~min}$, with very little effect on the postsynaptic muscle membrane potential or on the frequency or amplitude of the spontaneous mejps (Fig. 3A). At low doses of toxin (c. $10^{-10} \mathrm{M}$ ), release was nearly, but not completely, blocked. As shown in Figure 3, $B, C$, the amplitude of the ejp varied discontinuously between 0 and a value predicted accurately by the 


\section{A. Gel Filtration}
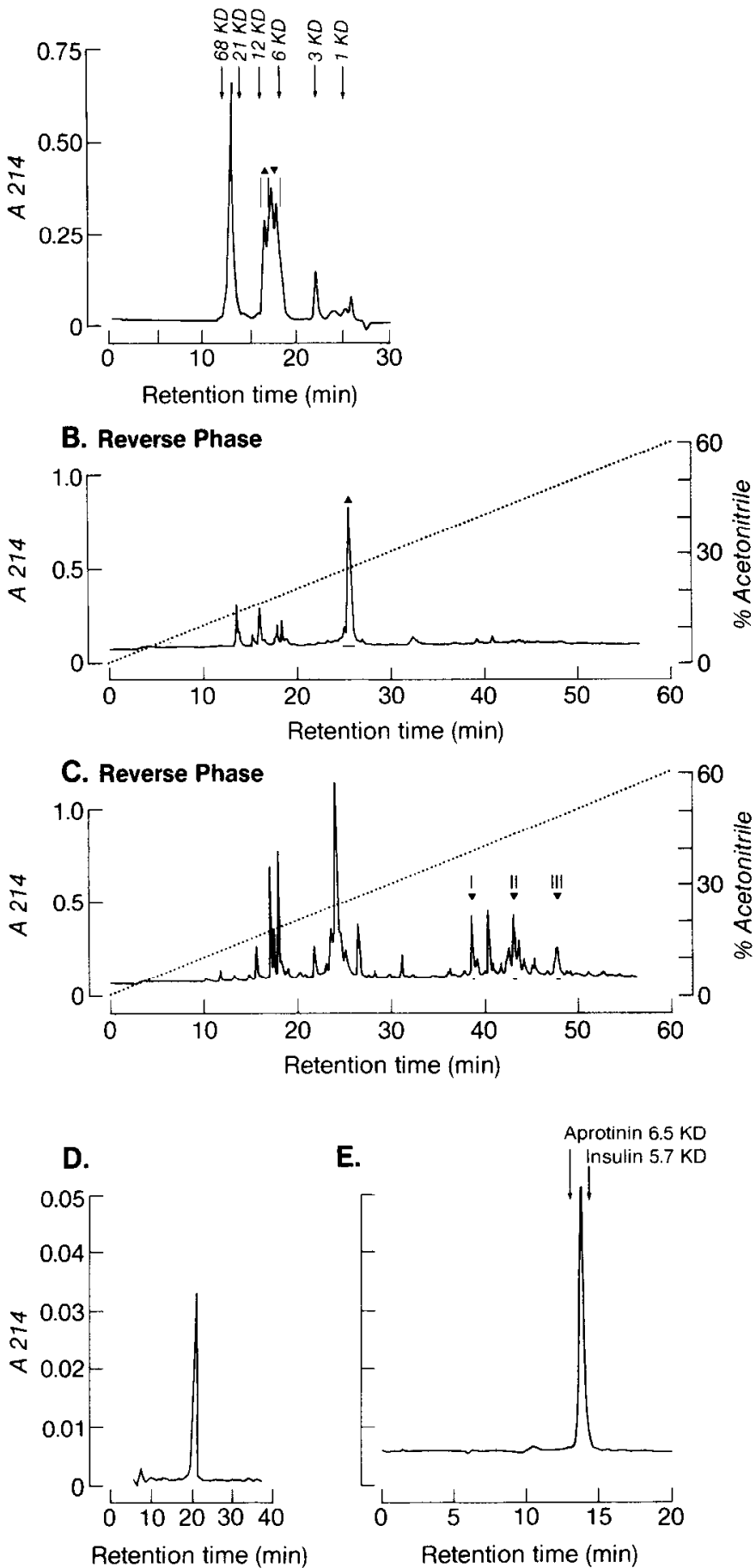

Figure 2. A, HPLC gel filtration separates crude venom into inhibitory $(\boldsymbol{\nabla})$ and excitatory $(\boldsymbol{\Delta})$ fractions, based on apparent $M_{\mathrm{r}}, B$, Gradient elution on reverse-phase HPLC of excitatory fractions from the gel filtration reveals a major peak of excitatory activity $(\mathbf{\Delta})$. $C$, Gradient elution on reverse-phase HPLC of inhibitory fractions from gel filtration reveals a region of inhibitory activity that includes 3 major peaks $(I$, $I I, I I I)$. Peak II was repurified in a second solvent system on reverse phase, and finally again on HPLC gel filtration under high-resolution conditions. Analytical traces of the final material in these last 2 systems are shown in $D$ (reverse phase) and $E$ (gel filtration). Arrows show the elution positions of insulin $(5.7 \mathrm{kDa})$ and aprotinin $(6.5 \mathrm{kDa})$.
A.
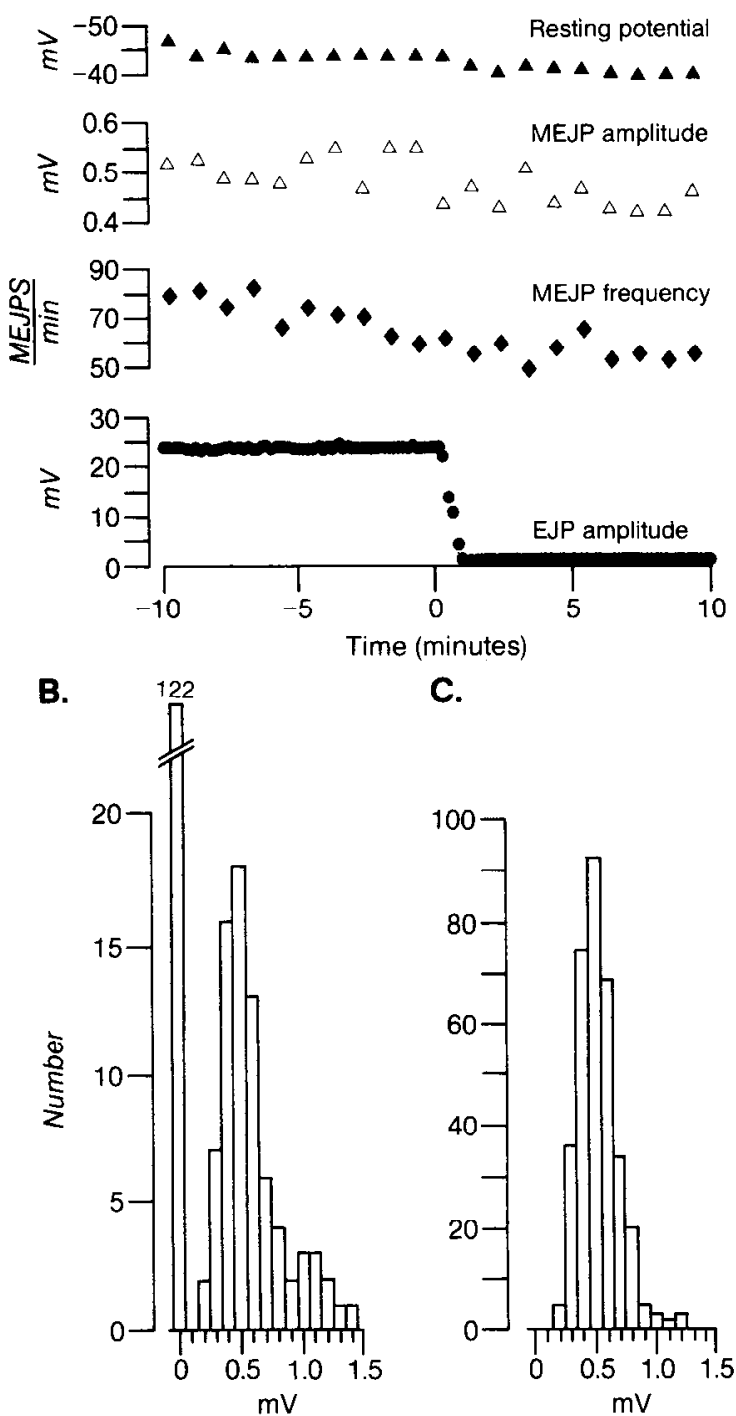

c.

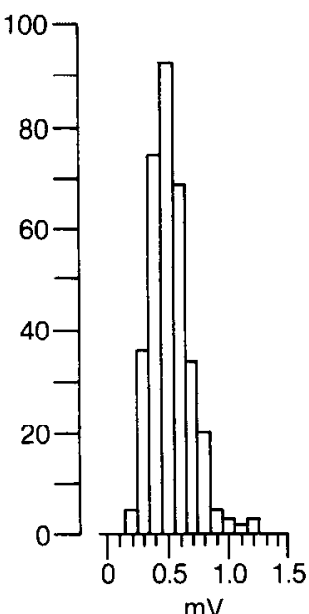

Figure 3. Alpha-PLTX II causes a specific reduction in the quantal content of the ejp. $A$, Effect of alpha-PLTX II on 4 parameters of synaptic transmission measured continuously during rapid and complete transmission block induced by application of $10^{-8} \mathrm{M}$ toxin. Toxin was applied at time 0 . The nerve was stimulated once every $10 \mathrm{sec}$. Effects on postsynaptic membrane potential $(\boldsymbol{\Lambda})$, miniature excitatory junction potential (mejp) amplitude $(\Delta)$, and mejp frequency $(\bullet)$ are small, while the ejp $(\Theta)$ is quickly and completely blocked. $B$ and $C$, The quantal nature of transmission is preserved during partial block of transmission induced by application of approximately $10^{-10} \mathrm{M}$ alpha-PLTX II for about 20 min. Mean quantal content was reduced from normal levels (greater than 40 ) to approximately 0.5 by application of $10^{-10} \mathrm{M}$ alphaPLTX II. A histogram of 200 nerve-evoked ejps, including 122 failures, is shown in $B$, and a histogram of 345 mejp amplitudes is shown in $C$. Mean quantal content, calculated by the method of failures, was 0.49 , and was 0.44 when calculated by dividing the mean ejp amplitude by the mean mejp amplitude. The distribution of the evoked potentials closely parallels the mejp amplitudes as expected at very low quantal content.

amplitudes of the spontaneous mejps; the quantal content was reduced from greater than 40 , before the application of toxin, to about 0.5 , demonstrating that the toxin acted by blocking transmitter release.

Alpha-PLTX II does not act by blocking nerve conduction. 
Figure 4. Recurrent terminal spikes in eag $S h^{\text {rk0 } 020}$ are not blocked by TTX. Top traces are extracellular recordings from a larval segmental nerve made near the nerve terminal. Bottom traces are intracellular recordings from the postsynaptic muscle. $a$, Characteristic train of terminal spikes (arrows) closely associated with the prolonged ejp. $b$, Residual spikes still associated with the ejp evoked by direct stimulation of the terminal in the presence of $10^{-5} \mathrm{M}$ TTX. (Calcium was $0.15 \mathrm{~mm}$.) a

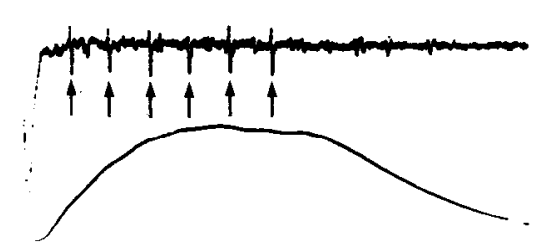

b TTX

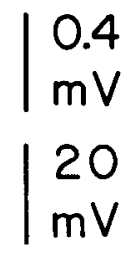

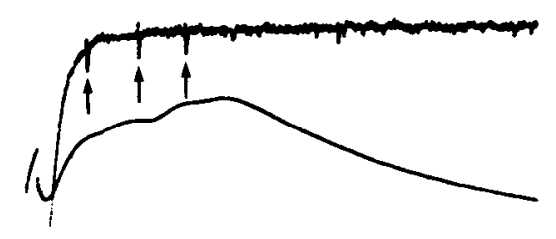

$\overline{20 \mathrm{~ms}}$

A

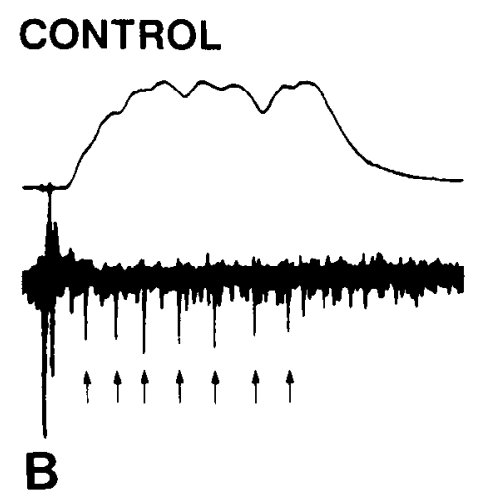

PLTX + 2 MIN

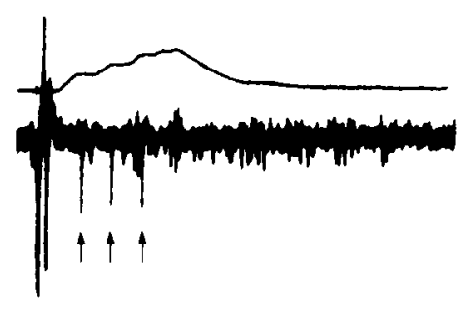

C

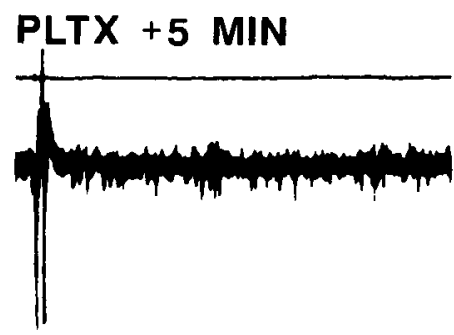

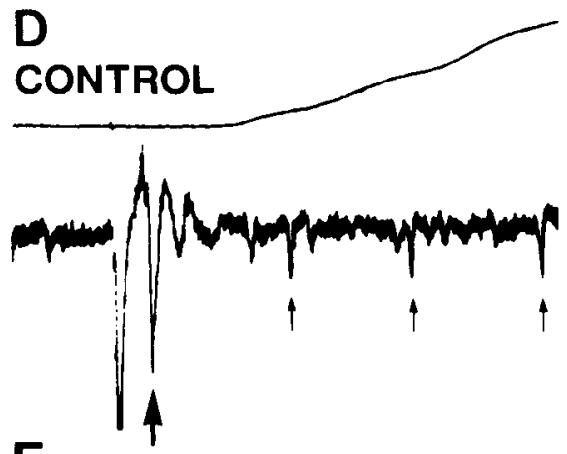

E

\section{PLTX}
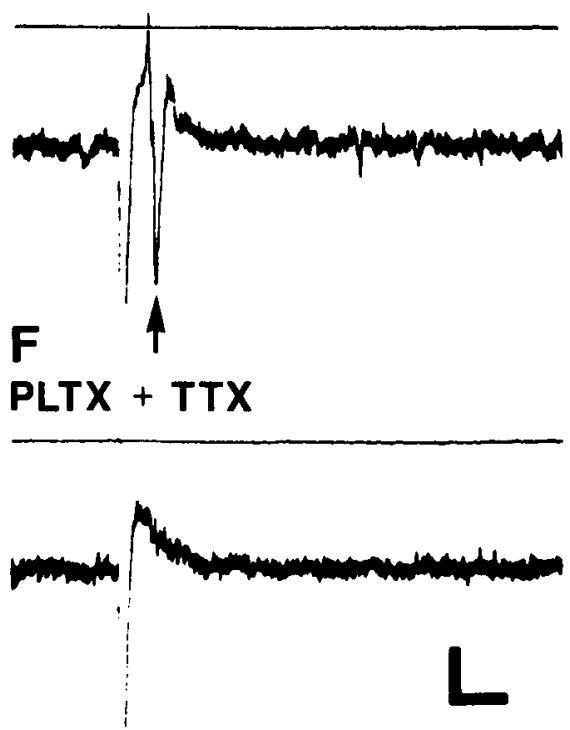

Figure 5. Alpha-PLTX II blocks electrical activity at or near the nerve terminal. $A-F$, Sets of simultaneous intracellular recordings (top traces) from the larval muscle, and extracellular recordings (bottom traces) from the larval nerve near the terminal region in an abnormally excitable mutant eag $S h^{\mathrm{r} \text { K0I20 }}$ in $0.15 \mathrm{~mm}$ calcium. $A$, Control traces showing a typical prolonged ejp associated with a train of extracellularly recorded spikes (small arrows) originating at or near the terminal. The number of these recurrent spikes correlates well with the duration of the ejp. $B$, Recordings made $2 \mathrm{~min}$ after application of $10^{-9} \mathrm{M}$ alpha-PLTX II. Note the reduced ejp and concomitantly reduced number of terminal spikes. $C$, Recording made after $5 \mathrm{~min}$ in the toxin. Both the ejp and terminal spikes arc abolishcd. $D-F$, Rccordings made at a faster time scale showing the presence or absence of the propagated compound action potential in the nerve (large arrow). $D$, Control traces before application of toxin. $E$, Recordings made 5 min after application of alpha-PLTX II. The compound action potential was still present after the ejp and associated terminal spikes were completely blocked. (The shape of the compound action potential showed small variations during an experiment, but there was no consistent alteration following the application of toxin.) $F$, Recordings made after application of $5 \times 10^{-6} \mathrm{M}$ TTX. TTX blocked the remaining compound action potential in the alpha-PLTX II-treated preparation, leaving only the stimulus artifact. Vertical calibration bar: $10 \mathrm{mV}$ (top traces); $0.2 \mathrm{mV}$ (bottom traces). Horizontal calibration bar: $20 \mathrm{msec}(A-C) ; 5 \mathrm{msec}(D-F)$. 
The toxin did not block the compound action potential recorded from the nerve (Fig. $5 E$ ). Furthermore, the toxin was still effective in blocking transmitter release when nerve conduction was blocked by TTX and the nerve terminal was depolarized directly by local stimulation (not shown). These results demonstrate that alpha-PLTX II blocks transmitter release without blocking nerve conduction. The toxin may block the entry of calcium into the nerve terminal. Alternatively, it may act upon subsequent biochemical processes involved in the release of transmitter.

Indirect evidence, obtained through the use of Drosophila mutants with defective potassium currents, suggests that alphaPLTX II probably blocks calcium entry into the nerve terminal. The ether a go go (eag) mutation produces flies with reduced delayed-rectifier currents, while the Shaker $(S h)$ mutation eliminates the transient $A$ current. Flies with both mutations thus have severely defective potassium conductances and therefore extremely excitable neuronal membranes (Salkof and Wyman, 1981; Ganetzky and Wu, 1982; Wu et al., 1983). In abnormally excitable mutants such as eag $S h^{\text {rK0120 }}$, a single nerve stimulus can cause a prolonged action potential in the nerve terminal and prolonged calcium-dependent release. The prolonged action potential originating at or near the motor nerve terminal is associated with recurrent spikes that can be recorded extracellularly via a suction electrode placed on the nerve near the terminal (Jan and Jan, 1979; Ganetzky and Wu, 1982) (Fig. 4a). When nerve conduction is blocked by TTX and the nerve terminal is stimulated directly, recurrent spikes are reduced in number, but still evident (Fig. $4 b$ ), suggesting that they arise at least in partial from a TTX-resistant mechanism (Jan and Jan, 1979). Since the recurrent spikes are blocked by Co or Cd, they are most likely associated with calcium currents. When alphaPLTX II was applied to the mutant neuromuscular preparation, the recurrent spikes were blocked simultaneously with the ejp (Fig. 5). Therefore, this toxin affects electrical properties of the nerve terminal. A likely target of the toxin would be the calcium channels in the presynaptic nerve terminal, although we cannot rule out the possibility that some other ionic mechanism is involved.

\section{Discussion}

Presynaptic toxins often show a unique specificity of action. Omega-Conotoxin, for example, is a potent blocker of neuronal calcium channels at the frog neuromuscular junction, has a complicated effect on mammalian CNS, and is inactive on both mouse and Drosophila neuromuscular junctions (Kerr and Yoshikami, 1984; Olivera et al., 1985). Apparently, alpha-PLTX II specifically affects the insect nerve terminal. Alpha-PLTX II has not been found to be active in vertebrates. For example, it does not block the frog neuromuscular junction at $10^{-8} \mathrm{M}$ concentration. In Drosophila, the toxin has no effect on the inward calcium or outward potassium currents of Drosophila pupal flight muscle, and it does not affect the divalent cation-dependent action potentials generated in larval muscle in the presence of $1 \mathrm{~mm} \mathrm{Sr}$. Therefore, this toxin does not block calcium channels in Drosophila muscle.

Hololena toxin has an action similar to that of alpha-PLTX II on fly neuromuscular junction, but is apparently unrelated to Plectreurys toxins biochemically (Bowers et al., 1987). Hololena toxin is a significantly larger molecule, apparently consisting of 2 disulfide-bonded peptides, rather than the single chain of PLTX. The 2 toxins behave quite differently on reverse-phase chro- matography, do not appear to share antigenic determinants, and preliminary cross-competition experiments in anatomical binding studies are consistent with separate binding sites. If, in fact, the binding sites are separate, it is possible that these toxins act through completely separate mechanisms. They might also simply act at separate sites on the same target molecule.

The venoms we have studied probably contain toxins that act on other targets and are specific for synapses in animals other than insects. A presynaptic toxin that appears to act selectively on certain vertebrate central synapses has recently been purified in another laboratory from the venom of Agclenopsis aperta (Jackson et al., 1986).

We suspect that the alpha-Plectreurys toxins are either potent blockers of insect presynaptic calcium channels or that they specifically affect processes closely linked to calcium entry at the nerve terminal. As such, they may be useful anatomical and biochemical probes of neurotransmission. The availability of a battery of presynaptic toxins with similar actions in different species and with different sites of action within species could contribute greatly to our understanding of the structure and function of the molecules involved in calcium-dependent release of neurotransmitter.

\section{References}

Agnew, W. S. (1984) Voltage-regulated sodium channel molecules. Annu. Rev. Physiol. 46: 517-530.

Anholt, R., J. Lindstrom, and M. Montal (1984) The molecular basis of neurotransmission: Structure and function of the nicotinic acetylcholine receptor. In Enzymes of Biological Membranes, vol. 3, A. Martonosi, ed., pp. 335-401, Plenum, New York.

Bowers, C. W., H. S. Phillips, P. Lee, Y. N. Jan, and L. Y. Jan (1987) The identification and purification of an irreversible presynaptic neurotoxin from the venom of the spider, Hololena curta. Proc. Natl. Acad. Sci. USA (in press).

Catterall, W. A. (1986) Molecular properties of voltage-sensitive sodium channels. Annu. Rev. Biochem. 55: 953-954.

Ganetzky, B., and C. F. Wu (1982) Indirect suppression involving behavioral mutants with altered nerve excitability in Drosophila melanogaster. Genetics 100: 597-614.

Halliwell, J. V., I. B. Othman, A. Pelchen-Matthews, and J. O. Dolly (1986) Central action of dendrotoxin: Selective reduction of a transient $K$ conductance in hippocampus and binding to localized acceptors. Proc. Natl. Acad. Sci. USA 83: 493-497.

Hughes, M., G. Romey, D. Duval, J. P. Vincent, and M. Lazadunski (1982) Apamin as a selective blocker of the calcium-dependent potassium channel in neuroblastoma cells: Voltage-clamp and biochemical charactcrization of the toxin reccptor. Proc. Natl. Acad. Sci. USA 79: 1308-1312.

Jackson, H., M. Urnes, W. R. Gray, and T. N. Parks (1986) Presynaptic blockage of transmission by a potent long lasting toxin from Agelenopsis aperta spiders. Soc. Neurosci. Abstr. 12: 730.

Jan, L. Y., and Y. N. Jan (1976) Properties of the larval neuromuscular junction in Drosophila melanogaster. J. Physiol. (Lond.) 262: 189214.

Jan, Y. N., and L. Y. Jan (1979) Genetic dissection of synaptic transmission in Drosophila melanogaster in insect neurobiology and pesticide actions. In Society of Chemical Industry, F. E. Rickett, ed., pp. 161-168, London.

Kerr, L. M., and D. Yoshikami (1984) A venom peptide with a novel presynaptic blocking action. Nature 308: 282-284.

Miller, C., E. Moczydlowski, R. Latorre, and M. Phillips (1985) Charabdotoxin, a protein inhibitor of single $\mathrm{Ca}^{2+}$-activated $\mathrm{K}^{+}$channels from mammalian skeletal muscle. Nature 313:316-318.

Noda, M., H. Takahashi, T. Tanabe, M. Toyosato, S. Kikyotani, Y. Furutani, T. Hirose, H. Takashima, S. Inayama, T. Miyata, and S. Numa (1983) Structural homology of Torpedo californica acetylcholine receptor subunits. Nature 302: 528-532.

Noda, M., T. Ikeda, H. Suzuki, H. Takashima, T. Takahashi, M. Kuno, and S. Numa (1986) Expression of functional sodium channels from cloned cDNA. Nature 322: 826-828. 
Olivera, B. M., W. R. Gray, R. Zeikus, J. M. McIntosh, J. Varga, J. Rivier, V. deSantos, and L. J. Cruz (1985) Peptide neurotoxins from fish-hunting cone snails. Science 230: 1338-1343.

Salkoff, L., and R. Wyman (1981) Genetic modification of potassium channels in Drosophila Shaker mutants. Nature 293: 228-230.

Wu, C. F., B. Ganetzky, L. Y. Jan, Y. N. Jan, and S. Benzer (1978) A
Drosophila mutant with a temperature-sensitive block in nerve conduction. Proc. Natl. Acad. Sci. USA 75: 4047-4051.

Wu, C. F., B. Ganetzky, F. N. Haughland, and A.-X. Liu (1983) Potassium currents in Drosophila: Different components affected by mutations of two genes. Science 220: 1076-1078. 